2009 mit drastischen Verlusten der Linken als Vorboten für den unaufhaltsamen Sieg der rechten Parteien bei den Parlamentswahlen von 2010 klar abzeichnete.

Ungarn hat also eine Regierung mit sehr breiter politischer und damit vermeintlich stabiler Unterstützung. Aber die Wirkungen der Wirtschaftskrise, die Verschuldung, die sozialen Probleme, Spannungen und Ungerechtigkeiten sind destabilisierende Faktoren geblieben. FIDESZ/KDNP hat große Ziele: Stabilisierung der Wirtschaft und Gesellschaft, Umformung des Sozial-, Gesundheits- und Bildungswesens, Stärkung der nationalen Einheit, ein starkes Ungarn mit europäischer Ausstrahlung im Jahr seiner Ratspräsidentschaft 2011. Diese können aber viele schwierige und zweischneidige Entscheidungen mit kontraproduktiven Wirkungen erzwingen, so dass es doch zu einer politischen Destabilisierung kommen könnte. Und: Stabilität ist nicht alles, Ungarn braucht auch eine „Demokratisierung der Demokratie“. Der ungarische Parlamentarismus und die demokratisch-politische Kultur bedürfen langfristiger weiterer Entwicklung. Es fehlen effiziente institutionelle Lösungen und kulturelle Muster der Vergangenheitsbewältigung und Konfliktlösung, wegen des fehlenden „Konsenses der Demokraten“.

\title{
Fraktionsprofile nach den Wahlen 2010 in Ungarn: Abschottung der parlamentarischen Elite?*
}

\author{
Sándor Kurtán und Gabriella Ilonszki
}

\section{Der Kontext}

Die Analyse der Profile von Parlamentsabgeordneten ist ein zentrales Thema der (empirischen) Repräsentationsforschung. Insbesondere in den letzen zwei Jahrzehnten, parallel zur Diskussion um eine Legitimationskrise der repräsentativen Demokratie, vervielfachten sich Ansätze und Studien, die konkret jene Frage beantworten wollten, inwieweit die gesellschaftlichen und politischen Eigenschaften der Abgeordneten den demokratischen Inhalt des Systems beeinflussen. Norris' und Lovenduskis Untersuchung war bahnbrechend in Bezug auf die soziodemographischen Gesichtspunkte, Andewegs und Thomassens Beitrag für bestimmte politische Karrieremuster. ${ }^{1}$ Heute kann es als wissenschaftliches Faktum gelten, dass der gesellschaftliche Hintergrund und der politische Karriereweg der Abgeordneten eng mit ihrer Tätigkeit zusammenhängen, also aus dem Blickwinkel des politischen Systems einen wichtigen Faktor darstellen, wobei hinzuzufügen ist, dass Maß und Art dieses Zusammenhanges noch durch weitere Studien detailliert belegt werden muss.

* Diese Studie wurde im Rahmen des TÁMOP-Projekts, Nr. 4.2.1.B-09/1/-KMR-2010-0005, angefertigt.

1 Vgl. Pippa Norris / Joni Lovenduski, Political Recruitment. Gender, Class and Race in the British Parliament, Cambridge 1995; Rudy B. Andeweg / Jacques J.A. Thomassen, Modes of Political Representation: Toward a New Typology, in: Legislative Studies Quarterly, 30. Jg. (2005), H. 4, S. $507-528$. 
Diese Fragen entfalten für die immer noch jungen Demokratien Ostmittel- und Osteuropas besondere Bedeutung. Daher soll es im Folgenden um das Profil der Parlamentsabgeordneten in Ungarn gehen, und zwar aus der Sicht der Rekrutierung und Selektion. Rekrutierung und Selektion müssen unterschieden werden ${ }^{2}$, hängen jedoch eng miteinander zusammen: Die Rekrutierung ist der Prozess der Gestaltung des politischen Nachwuchses, während die Auswahl der Kandidaten - die natürlich auf die Rekrutierung aufbaut - einen eingegrenzteren Prozess darstellt. Bei beiden werden sowohl gesellschaftliche als auch politische Aspekte sichtbar, aber bei der Selektion kommen die Standpunkte des Akteurs, also der Partei, eindeutiger und entschiedener zur Geltung. Daher wird das Profil der Parlamentsabgeordneten in erster Linie durch die Rekrutierungs- und Selektionsstrategien der Parteien bestimmt. Daher soll es hier - neben einer Übersicht über das gesamte Parlament - vor allem um die Darstellung der einzelnen Fraktionen gehen.

In der Praxis der europäischen Demokratien spielen Parteien die entscheidende Rolle bei der Auswahl der Kandidaten - und so in letzter Instanz auch bei der Auswahl der Abgeordneten. Für Ungarn muss diese Feststellung um zwei Aspekte ergänzt werden. (1) In Ungarn haben die Wähler keine Möglichkeit, durch offene Listen oder präferenzielle Stimmenabgabe die Reihenfolge der durch die Parteien bestimmten Kandidaten zu beeinflussen. Es verrät viel über den Zustand der Demokratie, dass bei den Plänen im Zusammenhang zur Reform des ungarischen Wahlsystems dieser Gesichtspunkt nie auftauchte. Sowohl die Mitglieder der politischen Elite als auch die sogenannte Experten/Ratgeber haben sich mit diesem Thema nur aus dem Blickwinkel der Besitzer der Machtpositionen und der zwischen ihnen bestehenden Vereinbarungen beschäftigt. Die Aktivität der Wähler sollte aber nicht bloß auf die Wahl zwischen Parteien reduziert werden. Um nur ein zeitlich und räumlich nahes Beispiel zu nennen: Durch die bei den tschechischen Wahlen des Jahres 2010 auf Facebook gestartete Initiative und den populären Slogan („Gib deine Stimme den letzten vier auf der Liste! Gib deine Stimme nicht Dinosauriern!") sanken der Anteil der Wiedergewählten von 56 auf 43 Prozent und der Anteil der Frauen um sieben Punkte auf 22 Prozent. Natürlich kann man sich über das optimale Verhältnis von „Altgedienten“ zu Neulingen streiten, da sich hier der Wert der Professionalisierung einerseits und die Angst um die politischen Folgen der sich von den Wählern entfernenden, sich „einbetonierenden“ Abgeordnetengruppe andererseits gegenüberstehen. Daran wird nicht zuletzt die Divergenz zwischen der parteigesteuerten Selektion und den Wählerpräferenzen deutlich, die durch Wahlregeln gelindert werden kann.

Im Falle Ungarns hätte dies eine besonders große Bedeutung, da das politische System von mehreren Autoren mit dem Begriff der overpartization, einer übertriebenen Parteiendominanz, charakterisiert wurde. ${ }^{3}$ Unter diesen Umständen hätten die Wähler nur durch

2 Vgl. Reuven Y. Hazan / Gideon Rahat, Democracy within Parties. Candidate Selection Methods and their Political Consequences, Oxford 2010; Reuven Y. Hazan, Candidate Selection. in: Lawrence LeDuc / Richard G. Niemi / Pippa Norris (Hrsg.), Comparing Democracies. New Challenges in the Study of Elections and Voting, London 2002, S. $108-126$.

3 Siehe dazu Attila Agh, The Hungarian Party System and Party Theory in the Transition in Central Europe, in: Journal of Theoretical Politics, 6. Jg. (1994), H. 2, S. 217 - 239; Enyedi Zsolt, Pártrendszer és politikai konszolidáció, in: Politikatudományi Szemle, 7. Jg. (1998), H. 3, S. 5 - 33; Tóka Gábor, Vezérek csodálói. A magyar választói magatartás nemzetközi összehasonlításban, in: Gergely Karácsony (Hrsg.), Parlamenti választás, Budapest 2006, S. 17 - 58; Enyedi Zsolt / Fernando Casal Bértoa, Pártverseny mintázatok Közép-Kelet-Európában, in: Politikatudományi Szemle, 19. Jg. (2010), H. 1, S. 7 - 33. 
die Öffnung der Listen eine stärkere Möglichkeit, den Einfluss der Parteien auf die Auswahl der Abgeordneten einzuschränken. Zwar existiert auch die direktere Wirkung der Personenstimme in den Einzelwahlkreisen ${ }^{4}$, aber ihre Durchsetzung wird durch das Gewicht der Parteien vermindert. ${ }^{5}$

(2) Neben der selbstverständlichen Akzeptanz der Tatsache, dass die repräsentative Demokratie parteiengesteuert ist, muss auf die starke Stellung der Parteien bei der Kandidatenaufstellung hingewiesen werden.

Das Instrument der Kandidatenaufstellung spiegelt einerseits den Charakter der Parteien wider, beeinflusst andererseits ihre Politik. ${ }^{6}$ Die Zentralisierung der Auswahl, wie sie in Ungarn anzutreffen ist, spielt nicht nur für die Leistungsfähigkeit von Parlament und Demokratie eine entscheidende Rolle, sondern - damit eng verbunden - auch für die Eigenschaften der Abgeordneten und wird vermutlich eine homogenisierende Wirkung nach sich ziehen: Die Abgeordneten werden sich immer mehr ähneln. Diese Fragen sind nur als Hintergrundfaktoren von Bedeutung, wirken aber bei der Formulierung des Profils der Abgeordneten mit und sind deshalb eine Erwähnung wert.

Die Eigenschaften der Abgeordneten geben über gesellschaftliche Prozesse Aufschluss. In diesem Zusammenhang ist der gut bekannte Ansatz wegweisend: Sowohl das Angebot als auch die Nachfrage bestimmen, wer Mitglied des Parlaments wird. Nicht nur die Präferenzen der Parteien, sondern auch ihre Selektionsmöglichkeiten haben Folgen. Warum gibt es zum Beispiel weniger Frauen im Parlament? Inwieweit ist dies auf verzerrende Effekte der Auswahl zurückzuführen und inwieweit auf den Mangel an Kandidaten? Auf diese Fragen kann nicht im Rahmen dieses Beitrages eingegangen werden, frühere Studien aus Ungarn berichten aber, dass nicht die geringe Zahl der Kandidaten den Grund der Unterrepräsentation der Frauen ausmacht. ${ }^{7}$

In den Kontext der Kandidatenaufstellung gehören auch die parlamentarischen Kriterien für das Profil der Abgeordneten. Dabei steht die Anpassung an den Professionalisierungsprozess und dessen Merkmale im Vordergrund. Untersucht man die Bedingungen der Wiederwahl, die errungenen Positionen und deren Zusammenhänge mit anderen KarriereEigenschaften der Abgeordneten ergibt sich die Möglichkeit, die Profile der Parlamentsfraktionen darzulegen - und damit kann das Verhältnis zwischen der Rationalität der parteigesteuerten Selektion und der parlamentarischen Rationalität erhellt werden.

Im Zusammenhang mit den Parlamentswahlen in Ungarn des Jahres 2010 heben die Analysen vor allem den Wandel des Parteiensystems hervor. ${ }^{8}$ Das Parteibündnis FIDESZMagyar Polgári Szövetség (FIDESZ-Ungarischer Bürgerbund) und KDNP (Kereszténydemokrata Néppárt / Christdemokratische Volkspartei) $)^{9}$ konnte eine Zweidrittelmehrheit der Mandate im Parlament erringen, während es zum endgültigen Niedergang der beiden gro-

4 Vgl. Bruce Cain / John Ferejohn / Morris Fiorina, The Personal Vote: Constituency Service and Electoral Independence, Cambridge 1987; John Carey / Matthew S. Shugart, Incentives to Cultivate a Personal Vote, in: Electoral Studies, 14. Jg. (1995), H. 4, S. 417 - 439.

5 Vgl. Zsófia Papp, A stabilitás forrásai a képviselők megválasztásában, in: Gabriella Ilonszki (Hrsg.), Amatőr és hivatásos politikusok. Képviselők Magyarországon II, Budapest 2008, S. 95 - 119.

6 Vgl. Reuven Y. Hazan / Gideon Rahat, a.a.O. (Fn. 2); Reuven Y. Hazan a.a.O. (Fn. 2).

7 Vgl. Gabriella Ilonszki / Várnagy Réka, Vegyes választási rendszer és női képviselet, in: Politikatudományi Szemle, 16. Jg. (2007), H. 1, S. 93 - 109.

8 Vgl. auch den Beitrag von Máté Szabó und Ágnes Lux in diesem Heft der ZParl.

9 Nachfolgend werden im Text die ungarischen Abkürzungen der Parteien verwendet. 


\begin{tabular}{|c|c|c|c|c|c|c|c|c|}
\hline \multicolumn{9}{|c|}{$\begin{array}{l}\text { Tabelle 1a: Zusammensetzung des ungarischen Parlaments im Hinblick auf Kontinuität und } \\
\text { Erneuerung }\end{array}$} \\
\hline \multirow[t]{2}{*}{ Partei } & \multicolumn{2}{|c|}{$\begin{array}{c}\text { Alte } \\
\text { Abgeordnete }^{1}\end{array}$} & \multicolumn{2}{|c|}{$\begin{array}{c}\text { Neue } \\
\text { Abgeordnete }^{2}\end{array}$} & \multicolumn{2}{|c|}{ Rückkehrer ${ }^{3}$} & \multicolumn{2}{|c|}{$\begin{array}{l}\text { Abgeordnete } \\
\text { insgesamt }\end{array}$} \\
\hline & $\mathrm{N}$ & $\%$ & $\mathrm{~N}$ & $\%$ & $\mathrm{~N}$ & $\%$ & $\mathrm{~N}$ & $\%$ \\
\hline FIDESZ & 120 & 52,9 & 94 & 41,4 & 13 & 5,7 & 227 & 100 \\
\hline KDNP & 20 & 55,6 & 16 & 44,4 & - & - & 36 & 100 \\
\hline MSZP & 52 & 88,1 & 6 & 10,2 & 1 & 1,7 & 59 & 100 \\
\hline Jobbik & - & - & 44 & 93,6 & 3 & 6,4 & 47 & 100 \\
\hline LMP & - & - & 15 & 93,8 & 1 & 6,3 & 16 & 100 \\
\hline Unabhängig & 1 & - & - & - & - & - & 1 & 100 \\
\hline Insgesamt & 193 & 50,0 & 175 & 45,3 & 18 & 4,7 & 386 & 100 \\
\hline \multicolumn{9}{|c|}{$\begin{array}{l}1 \text { Abgeordnete am Ende der Wahlperiode } 2006 \text { bis } 2010 . \\
2 \text { War noch nie Abgeordneter. } \\
3 \text { War schon einmal Abgeordneter, aber nicht am Ende der vorhergehenden Wahlperiode. } \\
\text { Quelle: Ungarische Abgeordneten-Datenbank, Elitenforschungszentrum, Institut für Politikwissenschaft, } \\
\text { Corvinus Universität Budapest. }\end{array}$} \\
\hline
\end{tabular}

ßen Systemwechselparteien MDF (Magyar Demokrata Fórum / Ungarisches Demokratisches Forum) und SZDSZ (Szabad Demokraták Szövetsége / Bund Freier Demokraten) kam. Die Nachfolgepartei MSZP (Magyar Szocialista Párt / Ungarische Sozialistische Partei) wurde drastisch geschwächt. Außerdem zogen zwei neue Parteien ins Parlament ein.

Diese Entwicklungen legen zwei Fragen nahe: (1) Inwieweit ähneln die Abgeordneten, die 2010 ins Parlament zogen, den früheren beziehungsweise in welchen Aspekten unterscheiden sie sich von ihnen? (2) Wie gestaltet sich der Beitrag der einzelnen Parteien zu den Unterschieden? Im Hintergrund dieser Fragen befinden sich all jenes Wissen und jene Erfahrungen, die durch die Forschung der letzten 20 Jahre begründet wurden. Die Analyse der ersten fünf Parlamente in Ungarn hat zum Beispiel gezeigt, dass die Abgeordneten sich immer ähnlicher werden, sowohl hinsichtlich ihres gesellschaftlichen Hintergrundes als auch ihrer politischen Karriere. Bedeutende Unterschiede ergaben sich eher bei der Gegenüberstellung von kleinen und großen Fraktionen - aber auch nur, weil die kleineren Fraktionen stärker die Professionalisierungsprozesse aufwiesen, da nur die großen Fraktionen sich Amateure erlauben können. ${ }^{10} \mathrm{Zu}$ untersuchen ist nun, ob diese Befunde auch für das sechste ungarische Parlament gültig sind.

Die Grundlage der Untersuchung bildet die Ungarische Abgeordneten-Datenbank. Diese umfasst alle Abgeordneten zwischen 1884 und 1947, sowie zwischen 1990 und 2010 und enthält in 44 Variablen Angaben zu den Personen (unter anderem Geschlecht, Geburtsjahr, Geburtsort, Schulbildung, Beruf, Typ des Mandats, Parteimitgliedschaft, Verbindung zu Zivilorganisationen, Regierungsmitgliedschaft, parlamentarische Funktionen, Position in der Parteiführung). ${ }^{11}$

10 Vgl. Gabriella Ilonszki, Képviselők és képviselet Magyarországon a 19. és a 20. században, Budapest 2009, S. $163 \mathrm{ff}$.

11 Die Datenbank ist als CD-Beilage zu Gabriella Ilonszki (Hrsg.), a.a.O. (Fn. 5), publiziert worden (mit Ausnahme der Abgeordneten des 2010 gewählten Parlaments). 


\begin{tabular}{|c|c|c|c|c|c|c|c|c|c|c|c|c|}
\hline \multicolumn{13}{|c|}{ Tabelle 1b: Neue Abgeordnete nach Parlamentsfraktionen, 1990 bis 2010} \\
\hline \multirow[t]{2}{*}{ Partei } & \multicolumn{2}{|c|}{$\begin{array}{c}1990-1994 \\
(\mathrm{~N}=415)^{*}\end{array}$} & \multicolumn{2}{|c|}{$\begin{array}{c}1994-1998 \\
(\mathrm{~N}=402)^{*}\end{array}$} & \multicolumn{2}{|c|}{$\begin{array}{c}1998-2002 \\
(\mathrm{~N}=397)^{*}\end{array}$} & \multicolumn{2}{|c|}{$\begin{array}{c}2002-2006 \\
(\mathrm{~N}=412)^{*}\end{array}$} & \multicolumn{2}{|c|}{$\begin{array}{c}2006-2010 \\
(\mathrm{~N}=406)^{*}\end{array}$} & \multicolumn{2}{|c|}{$\begin{array}{c}2010- \\
(\mathrm{N}=386)^{*}\end{array}$} \\
\hline & $\mathrm{N}$ & $\%$ & $\mathrm{~N}$ & $\%$ & $\mathrm{~N}$ & $\%$ & $\mathrm{~N}$ & $\%$ & $\mathrm{~N}$ & $\%$ & $\mathrm{~N}$ & $\%$ \\
\hline FIDESZ & 26 & 100 & 6 & 27,3 & 114 & 76,5 & 40 & 23,1 & 47 & 31,5 & 94 & 41,4 \\
\hline FKGP & 41 & 91,1 & 21 & 75,0 & 34 & 65,4 & - & - & - & - & - & - \\
\hline Jobbik & - & - & - & - & - & - & - & - & - & - & 44 & 93,6 \\
\hline KDNP & 21 & 95,5 & 13 & 56,5 & - & - & - & - & 3 & 12,0 & 16 & 44,4 \\
\hline LMP & - & - & - & - & - & - & - & - & - & - & 15 & 93,8 \\
\hline MIÉP & - & - & - & - & 9 & 64,3 & - & - & - & - & - & - \\
\hline MDF & 162 & 93,6 & 13 & 34,2 & 9 & 52,9 & 7 & 29,2 & 3 & 25,1 & - & - \\
\hline MSZP & 35 & 81,4 & 185 & 84,9 & 26 & 18,9 & 87 & 45,8 & 54 & 27,6 & 6 & 10,2 \\
\hline SZDSZ & 96 & 98,0 & 18 & 25,7 & 3 & 11,5 & 4 & 17,4 & 8 & 34,8 & & \\
\hline Andere & 4 & 55,6 & 2 & 66,7 & - & - & - & - & 1 & 100 & - & - \\
\hline Insgesamt & 385 & 92,8 & 258 & 64,2 & 195 & 49,1 & 140 & 34,0 & 116 & 28,6 & 175 & 45,3 \\
\hline \multicolumn{13}{|c|}{$\begin{array}{l}\text { * N zeigt an, wie viele Abgeordnete insgesamt während einer Wahlperiode im Parlament dienten. } \\
\text { Quelle: Gabriella Ilonszki, Konszolidáció vagy bezárkózás? A 2006-ban megválasztott parlamenti képvi- } \\
\text { selők jellemzői, in: Gergely Karácsony (Hrsg.), Parlamenti választás, Budapest 2006, S. } 229 \text { - 252; Unga- } \\
\text { rische Abgeordneten-Datenbank. }\end{array}$} \\
\hline
\end{tabular}

\section{Die Zusammensetzung des Parlaments}

Das Wahlergebnis 2010 hatte zur Folge, dass im Parlament eine bedeutende Umstrukturierung vor sich ging: 45 Prozent der Abgeordneten sind neu. Auf den ersten Blick ist dieser Prozentsatz beträchtlich, erreicht aber weder die Erneuerungsrate nach dem Wahlsieg der MSZP im Jahre 1994 (64 Prozent) noch jene nach dem Vorstoß von FIDESZ 1998 (49 Prozent), ist aber zweifellos höher als jene der Wahlen 2002 und 2006 (siehe Tabelle 1a und 1b). Auch 2010 finden sich Abgeordnete, die nach einer Pause von mehreren Jahren erneut ins Parlament eingezogen sind. Diese „Rückkehrer“ machen zwar nicht einmal fünf Prozent der Abgeordneten aus, sind aber vor allem bei FIDESZ Indikator für ein Selektionskriterium.

Tabelle 2 zeigt die Zusammensetzung des Parlaments nach Fraktionen sowie nach Ursprung des Mandats. Man sieht, dass mit drei Ausnahmen in allen Einzelwahlkreisen die gemeinsame Liste von FIDESZ und KDNP gesiegt hat. ${ }^{12}$ So bekamen die Oppositionsparteien praktisch nur Listenmandate. Die Kandidaten von Jobbik (Jobbik Magyarországért / Für ein Besseres Ungarn) und LMP (Lehet Más a Politika / Politik kann anders sein) konnten in keinem Einzelwahlkreis einen Sieg erringen.

Diese Zusammensetzung des Parlaments, die die Veränderungen des Parteiensystems widerspiegelt, sagt noch nichts darüber aus, ob auch eine Erneuerung des Profils der Abgeordneten stattgefunden hat. Mit den Daten der Tabelle 3 werden die 2010 ausgeschiedenen Abgeordneten mit den Parlamentsneulingen verglichen und der Frage nachgegangen, welche charakteristischen Züge die Erneuerung aufweist. Wiederrum findet sich ein Beweis dafür, dass der Stuhl unter den Frauen im Parlament eher wackelt als bei Männern (obwohl es im

12 Zum ungarischen Wahlsystem siehe den Beitrag von Máté Szabó und Ágnes Lux in diesem Heft. 


\begin{tabular}{|l|c|c|c|c|c|}
\hline \multirow{2}{*}{ Tabelle 2: Zusammensetzung des ungarischen Parlaments 2010} \\
\hline \multirow{2}{*}{ Partei } & \multicolumn{2}{|c|}{ Abgeordnete } & \multicolumn{3}{c|}{ Typ des Mandats } \\
\cline { 2 - 6 } & Anzahl & $\%$ & Einzelwahlkreis & Territorialliste & Landesliste \\
\hline FIDESZ & 227 & 58,8 & 153 & 71 & 3 \\
KDNP & 36 & 9,3 & 20 & 16 & - \\
MSZP & 59 & 15,3 & 2 & 28 & 29 \\
Jobbik & 47 & 12,2 & - & 26 & 21 \\
LMP & 16 & 4,1 & - & 5 & 11 \\
Unabhängig & 1 & 0,3 & 1 & - & - \\
\hline Insgesamt & 386 & 100,0 & 176 & 146 & 64 \\
\hline Quelle: Ungarische Abgeordneten-Datenbank. \\
\hline
\end{tabular}

\begin{tabular}{|c|c|c|c|c|}
\hline & \multicolumn{2}{|c|}{$\begin{array}{l}\text { nach den Wahlen } 2010 \text { nicht } \\
\text { mehr im Parlament }(\mathrm{N}=186)\end{array}$} & \multicolumn{2}{|c|}{$\begin{array}{c}2010 \text { neu im Parlament } \\
(\mathrm{N}=175)\end{array}$} \\
\hline & $\mathrm{N}$ & $\%$ & $\mathrm{~N}$ & $\%$ \\
\hline Frauen & 28 & 15,1 & 21 & 11,9 \\
\hline Geburtsort Budapest & 45 & 24,2 & 53 & 30,3 \\
\hline Jurist oder Ökonom & 68 & 36,6 & 46 & 26,3 \\
\hline Führende Position in der Partei & 57 & 30,6 & 67 & 38,3 \\
\hline $\begin{array}{l}\text { Zur Zeit der Wahl } \\
\text { Kommunalpolitiker }\end{array}$ & 99 & 53,2 & 119 & 68,0 \\
\hline Durchschnittsalter & \multicolumn{2}{|c|}{52,0} & \multicolumn{2}{|c|}{42,9} \\
\hline
\end{tabular}

Tabelle 4: Sozio-demographische Eigenschaften der Abgeordneten, 1990 bis 2010

\begin{tabular}{|c|c|c|c|c|c|c|c|c|c|c|c|c|}
\hline & \multicolumn{2}{|c|}{$\begin{array}{c}1990-1994 \\
(\mathrm{~N}=415)\end{array}$} & \multicolumn{2}{|c|}{$\begin{array}{c}1994-1998 \\
(\mathrm{~N}=402)\end{array}$} & \multicolumn{2}{|c|}{$\begin{array}{c}1998-2002 \\
(\mathrm{~N}=397)\end{array}$} & \multicolumn{2}{|c|}{$\begin{array}{c}2002-2006 \\
(\mathrm{~N}=412)\end{array}$} & \multicolumn{2}{|c|}{$\begin{array}{c}2006-2010 \\
(\mathrm{~N}=406)\end{array}$} & \multicolumn{2}{|c|}{$\begin{array}{c}2010- \\
(\mathrm{N}=386)\end{array}$} \\
\hline & $\mathrm{N}$ & $\%$ & $\mathrm{~N}$ & $\%$ & $\mathrm{~N}$ & $\%$ & $\mathrm{~N}$ & $\%$ & $\mathrm{~N}$ & $\%$ & $\mathrm{~N}$ & $\%$ \\
\hline Frauen & 32 & 7,7 & 44 & 10,9 & 31 & 7,8 & 40 & 9,7 & 43 & 10,6 & 35 & 9,1 \\
\hline Geburtsort Budapest & 106 & 25,7 & 93 & 23,1 & 89 & 22,4 & 94 & 22,8 & 101 & 24,9 & 105 & 27,2 \\
\hline Hochschulabschluss & 375 & 90,8 & 362 & 90.0 & 368 & 92,7 & 370 & 93,9 & 373 & 91,9 & 336 & 87.0 \\
\hline Jurist & 89 & 21,4 & 77 & 19,2 & 87 & 21,9 & 84 & 20,9 & 84 & 20,7 & 79 & 20,5 \\
\hline Ökonom & 59 & 14,2 & 75 & 18,9 & 77 & 19,4 & 81 & 19,7 & 82 & 20,2 & 56 & 14,5 \\
\hline $\begin{array}{l}\text { Humanwissen- } \\
\text { schaften }\end{array}$ & 126 & 30,4 & 91 & 23,0 & 88 & 22,2 & 106 & 26,4 & 106 & 28,5 & 120 & 31,0 \\
\hline $\begin{array}{l}\text { Technische und } \\
\text { Agrarwissenschaften }\end{array}$ & 97 & 23,4 & 114 & 28,4 & 128 & 32,3 & 113 & 28,2 & 107 & 26,4 & 89 & 22,1 \\
\hline Medizin & 55 & 13,3 & 26 & 6,5 & 25 & 6,3 & 23 & 5,7 & 30 & 7,4 & 20 & 5,2 \\
\hline Andere & 20 & 4,8 & 21 & 5,3 & 33 & 8,3 & 32 & 8,0 & 41 & 10,1 & 12 & 3,1 \\
\hline Durchschnittsalter & \multicolumn{2}{|c|}{45,8} & \multicolumn{2}{|c|}{46,6} & \multicolumn{2}{|c|}{46,3} & \multicolumn{2}{|c|}{46,7} & \multicolumn{2}{|c|}{49,6} & \multicolumn{2}{|c|}{47,3} \\
\hline
\end{tabular}


ungarischen Parlament nur Stuhlreihen gibt); des Weiteren, dass die Anzahl der in Budapest geborenen unter den Neuen höher liegt, dass sie im Durchschnitt zehn Jahre jünger sind, als jene die nicht mehr gewählt wurden und in ihrem Kreis etwa zehn Prozent weniger Juristen und Ökonomen zu finden sind. Da diese für die Professionalisierung als besonders wichtig angesehen werden ${ }^{13}$, lassen die Daten darauf schließen, dass sich hier die Umrisse eines we-

\begin{tabular}{|c|c|c|c|c|c|c|c|c|c|c|c|c|}
\hline & \multicolumn{2}{|c|}{$\begin{array}{l}\text { FIDESZ } \\
(\mathrm{N}=227)\end{array}$} & \multicolumn{2}{|c|}{$\begin{array}{l}\mathrm{KDNP} \\
(\mathrm{N}=36)\end{array}$} & \multicolumn{2}{|c|}{$\begin{array}{l}\text { MSZP } \\
(\mathrm{N}=59)\end{array}$} & \multicolumn{2}{|c|}{$\begin{array}{l}\text { Jobbik } \\
(\mathrm{N}=47)\end{array}$} & \multicolumn{2}{|c|}{$\begin{array}{l}\text { LMP } \\
(\mathrm{N}=16)\end{array}$} & \multicolumn{2}{|c|}{$\begin{array}{l}\text { Parlament } \\
\text { insgesamt } \\
(\mathrm{N}=386)\end{array}$} \\
\hline & $\mathrm{N}$ & $\%$ & $\mathrm{~N}$ & $\%$ & $\mathrm{~N}$ & $\%$ & $\mathrm{~N}$ & $\%$ & $\mathrm{~N}$ & $\%$ & $\mathrm{~N}$ & $\%$ \\
\hline Frauen & 20 & 8,8 & 2 & 5,6 & 5 & 8,5 & 3 & 6,4 & 5 & 31,3 & 35 & 9,1 \\
\hline Geburtsort Budapest & 54 & 23,8 & 10 & 27,8 & 14 & 23,7 & 17 & 36,2 & 10 & 62,5 & 105 & 27,2 \\
\hline Hochschulabschluss & 200 & 88,1 & 34 & 94,5 & 56 & 94,8 & 33 & 70,2 & 13 & 81,3 & 336 & 87,0 \\
\hline Jurist & 48 & 21,1 & 10 & 27,8 & 13 & 22,0 & 5 & 10,6 & 3 & 18,8 & 79 & 20,5 \\
\hline Ökonom & 30 & 13,2 & 4 & 11,1 & 16 & 27,1 & 5 & 10,6 & 1 & 6,3 & 56 & 14,5 \\
\hline $\begin{array}{l}\text { Humanwissen- } \\
\text { schaften }\end{array}$ & 70 & 30,9 & 11 & 30,5 & 17 & 28,8 & 13 & 27,7 & 9 & 56,3 & 120 & 31,0 \\
\hline $\begin{array}{l}\text { Technische und } \\
\text { Agrarwissen- } \\
\text { schaften }\end{array}$ & 55 & 23,8 & 6 & 16,7 & 14 & 23,7 & 4 & 8,6 & 2 & 12,5 & 85 & 22,1 \\
\hline Medizin & 14 & 6,2 & 3 & 8,3 & 1 & 1,7 & 2 & 4,7 & - & - & 20 & 5,2 \\
\hline Andere & 8 & 3,5 & - & - & 4 & 6,8 & - & - & - & - & 12 & 3,1 \\
\hline Durchschnittsalter & \multicolumn{2}{|c|}{47,8} & \multicolumn{2}{|c|}{55,8} & \multicolumn{2}{|c|}{49,4} & \multicolumn{2}{|c|}{40,5} & \multicolumn{2}{|c|}{37,3} & \multicolumn{2}{|c|}{47,3} \\
\hline $\begin{array}{l}\text { Wie oft gewählt } \\
\text { (Durchschnitt) }\end{array}$ & \multicolumn{2}{|c|}{2,3} & \multicolumn{2}{|c|}{2,3} & \multicolumn{2}{|c|}{3,4} & \multicolumn{2}{|c|}{1,1} & \multicolumn{2}{|c|}{1,1} & \multicolumn{2}{|c|}{2,3} \\
\hline $\begin{array}{l}\text { Führende Position } \\
\text { in der Partei }\end{array}$ & 65 & 28,6 & 14 & 38,9 & 27 & 45,9 & 24 & 51,1 & 5 & 31,3 & & \\
\hline $\begin{array}{l}\text { Bei der Wahl zum } \\
\text { Parlament gleichzei- } \\
\text { tig auch Kommunal- } \\
\text { politiker }\end{array}$ & 161 & 70,9 & 17 & 47,2 & 27 & 45,8 & 6 & 12,7 & 1 & 6,3 & 216 & 66,0 \\
\hline $\begin{array}{l}\text { Nach der Wahl } \\
\text { Posten in der } \\
\text { Regierung }\end{array}$ & 22 & 9,6 & 3 & 8,4 & - & - & - & - & - & - & 28 & 7,2 \\
\hline $\begin{array}{l}\text { Nach der Wahl } \\
\text { Posten im Parla- } \\
\text { ment }^{2}\end{array}$ & 6 & 2,6 & 2 & 5,6 & 3 & 5,1 & 3 & 6,4 & 1 & 6,3 & 16 & 4,2 \\
\hline $\begin{array}{l}\text { Nach der Wahl } \\
\text { Posten in einem } \\
\text { Ausschuss }{ }^{3}\end{array}$ & 11 & 4,8 & 6 & 16,7 & 3 & 5,1 & 2 & 4,3 & 1 & 6,3 & 19 & 4,9 \\
\hline \multicolumn{13}{|c|}{$\begin{array}{l}1 \text { Posten in der Regierung: Minister, Staatssekretäre. } \\
2 \text { Posten im Parlament: Präsident, Vizepräsidenten, Schriftführer. } \\
3 \text { Posten im Ausschuss: Vorsitz in einem ständigen Ausschuss. } \\
\text { Quelle: Ungarische Abgeordneten-Datenbank. }\end{array}$} \\
\hline
\end{tabular}

13 Vgl. Maurizio Cotta / Heinrich Best, Between Professionalization and Democratization. A Synoptic View on the Making of the European Representative, in: dies. (Hrsg.), Parliamentary Representatives in Europe 1848-2000, Oxford 2002, S. 493 - 526. 
niger professionellen Parlaments abzeichnen. Dem widersprechen aber nachdrücklich zwei politische Variablen: der kommunalpolitische Hintergrund und die führende Position innerhalb der Parteien. In beiden Fällen ist der Anteil jener, die solche Positionen unter den neuen Abgeordneten besitzen, bedeutend höher (im ersten Fall um elf, im zweiten um 15 Prozent). Das heißt aber, dass neben den Änderungen bei den soziodemographischen Eigenschaften die Machtkonzentration in der parlamentarischen Elite weitergeht.

\section{Eigenschaften der Abgeordneten nach Fraktionen}

Es liegt nahe, dass die „alten“ Fraktionen die alten Trends, das heißt die Professionalisierung und Positionshäufung weiterführen, während die beiden neuen Fraktionen auch in dieser Hinsicht etwas Neues aufzeigen.

\subsection{Die alt-neue FIDESZ}

FIDESZ errang ihren größten Sieg seit 1990. Die mit der KDNP gemeinsam aufgestellte Liste gewann über Zweidrittel aller Parlamentsmandate, mit der Folge, dass in großer Anzahl neue FIDESZ- (und christdemokratische) Abgeordnete ins Parlament gelangten. In der 227 Personen zählenden Fraktion sind 94 Neulinge, 120 repräsentierten die Kontinuität, und 13 sind Rückkehrer, das heißt solche Abgeordnete, die in einer früheren Wahlperiode schon einmal im Parlament saßen. Vergleicht man diese Gruppen, so ist festzustellen, dass sich die neuen FIDESZ-Abgeordneten in folgenden Zügen unterscheiden (vgl. Tabelle 6):

- Ihr Durchschnittsalter ist geringer, sowohl der Abgeordneten der vorhergehenden Wahlperiode als auch der Wiedergewählten (das ist nicht verwunderlich, denn allgemein sind neu Eintretende jünger);

- unter ihnen ist der Anteil mit Hochschulabschluss um sechs Prozentpunkte geringer als bei den „Alten“;

- der Anteil der Abgeordneten mit Wirtschaftsausbildung ist zurückgegangen;

- der Anteil der Absolventen von Humanwissenschaften ist bedeutend höher als zwischen 2006 und 2010; das gilt aber auch für die „Alten“, so dass dies für die ganze Fraktion kennzeichnend geworden ist (damit erinnert dieses Parlament an das 1990 gewählte, siehe dazu Tabelle 4);

- unter den neuen Abgeordneten ist der Anteil jener, die einen kommunalpolitischen Hintergrund besitzen, äußerst hoch (93 Prozent), was darauf hindeutet, dass die Tätigkeit auf lokaler Ebene (Gemeinde, Stadt, Komitat) als Karrierestation immer bedeutender wird.

Wenn eine Partei einen großen Sieg erringt, bedeutet dies einerseits, dass viele neue Politiker ins Parlament einziehen, andererseits eröffnet es aber auch die Chance der Wiederkehr für jene, die bei einer früheren Wahl ihren Sitz verloren haben. Zwar kann hier nicht auf die Mechanismen der Kandidatenaufstellung der einzelnen Parteien eingegangen werden; es ist aber klar, dass diese - neben anderen Faktoren - bestimmen, wer von den früheren Verlierern wieder kandidieren wird. An dieser Stelle kann nur registriert werden, ob es solche Personen gibt und wer sie sind.

Diese Art von Rückkehr taucht interessanterweise nach 1990 immer wieder im ungarischen Parlament auf, wenn eine Partei große Gewinne erzielt und ihre Fraktion zunimmt 


\begin{tabular}{|c|c|c|c|c|}
\hline \multirow[t]{2}{*}{ Tabelle 6: Charakteristika de } & \multirow[b]{2}{*}{$\begin{array}{l}\text { FIDESZ- } \\
\text { Fraktion, } \\
2006-2010 \\
(\mathrm{~N}=149)\end{array}$} & \multirow[b]{2}{*}{$\begin{array}{l}\text { FIDESZ- } \\
\text { Fraktion insge- } \\
\text { samt, 2010- } \\
(\mathrm{N}=227)\end{array}$} & \multirow[b]{2}{*}{$\begin{array}{l}\text { FIDESZ- } \\
\text { Fraktion Wie- } \\
\text { dergewählte, } \\
2010- \\
(\mathrm{N}=133)^{1}\end{array}$} & \multirow{2}{*}{$\begin{array}{c}\text { bis } 2010 \text { und } \\
\text { FIDESZ- } \\
\text { Fraktion neue } \\
\text { Abgeordnete, } \\
2010- \\
(\mathrm{N}=94)\end{array}$} \\
\hline & & & & \\
\hline Regierungsmitglied & - & 7,9 & 13,7 & - \\
\hline $\begin{array}{l}\text { Führende Position in einem } \\
\text { Parlamentsausschuss }\end{array}$ & 4,7 & 7,9 & 13,7 & 2,1 \\
\hline Führende Position im Parlament & 3,3 & 3,5 & 6,0 & 2,2 \\
\hline Führende Position in der Partei & 16,1 & 28,6 & 26,5 & 31,9 \\
\hline Geburtsort Budapest & 25,5 & 23,8 & 24,2 & 22,3 \\
\hline Hochschulabschluss & 88,6 & 88,1 & 90,2 & 84,1 \\
\hline Jurist & 19,5 & 21,1 & 23,5 & 17,9 \\
\hline Ökonom & 19,5 & 13,2 & 17,4 & 7,4 \\
\hline Humanwissenschaften & 18,3 & 30,9 & 31,1 & 30,5 \\
\hline $\begin{array}{l}\text { Technische und Agrarwissen- } \\
\text { schaften }\end{array}$ & 26,8 & 23,8 & 25,7 & 22,2 \\
\hline Medizin & 8,7 & 6,2 & 6,8 & 5,3 \\
\hline Andere & 4,7 & 3,5 & 6,1 & - \\
\hline Durchschnittsalter & 47,5 & 47,8 & 49,7 & 45,1 \\
\hline
\end{tabular}

(1998: 17 Personen, 2002: 18 Personen, 2006: acht Personen). ${ }^{14}$ Die Besonderheit beim FIDESZ-Sieg im Jahre 1998 war, dass die „Rückkehrer“ aus anderen Parteien kamen, während MSZP vier Jahre später die eigenen Leute zurückholte. 2010 zeigte aber auch FIDESZ ein anderes Bild. In der neugebildeten Fraktion sind 13 Personen (circa sechs Prozent), die zwischen 2006 und 2010 keine Abgeordneten waren. Zehn von ihnen hatten nur eine Wahlperiode dem Parlament nicht angehört, drei für zwei Perioden. Drei Abgeordnete begannen ihre Parlamentskarriere in einer anderen Partei (je einer in der FKGP, im SZDSZ und MDF.) Wenn es sich auch nur um drei Personen handelt, zeichnet sich doch ein Muster ab: Eintritt in FIDESZ aus einer anderen Partei, eine Wahlperiode wird ausgesetzt, und dann erst kommt die Möglichkeit einer erneuten Kandidatur.

Die Rückkehr in die Wirtschaft oder ins Berufsleben ist selten, die Rückkehrer waren alle während der ausgelassenen Wahlperiode aktiv in der Partei oder als Kommunalpolitiker des FIDESZ tätig, und zwar in der kommunalen Selbstverwaltung, auf Komitatsebene oder in der Hauptstadt, teilweise sogar als Vizebürgermeister. Die Karriere-Formel ist eindeutig: Der rückkehrende Abgeordnete war zwischen 2002 und 2006 im Parlament und gleichzeitig in der Kommunalpolitik tätig. ${ }^{15}$ Bei den Kommunalwahlen im Herbst 2006 (die

14 Vgl. Máté Gyömöre, A kiesők és a visszakerülők csoportjai a magyar országgyűlésben, in: Gabriella Ilonszki (Hrsg.), a.a.O. (Fn. 5), S. 121 - 152, S. 135.

15 In Ungarn darf seit 1994 ein Mitglied des Parlaments zugleich Bürgermeister sein. 
FIDESZ gewann) konnte er seine Position auf kommunaler Ebene behalten und blieb so nach dem Verlust des Parlamentsmandates weiterhin politisch aktiv. Und mit diesem Hintergrund konnte er es schaffen, 2010 erneut als Kandidat aufgestellt zu werden.

Wie ist die FIDESZ-Fraktion innerlich strukturiert, wenn das Augenmerk auf die möglichen politischen Positionen und deren Häufung gelenkt wird? Dabei sind folgende Positionen in Betracht zu ziehen: (1) leitende Funktion im Parlament; (2) leitende Funktion in einem ständigen Ausschuss; (3) Mitgliedschaft in der Regierung (Minister oder Staatssekretär); (4) führende Position in der Partei; (5) Position in der Kommunalpolitik. Da ein Regierungsmitglied keine Funktion im Parlament oder in einem Ausschuss ausüben kann, kann ein Abgeordneter maximal vier Positionen anhäufen. Diesen maximalen Wert hat keiner erreicht - was in einer solch großen Fraktion nicht verwunderlich ist. Aber ein Drittel der Fraktion hat zwei oder drei Funktionen inne ${ }^{16}$, während 11,5 Prozent aber überhaupt keine herausragende Position bekleiden und 55,5 Prozent nur eine. All dies hängt mit der Dienstzeit zusammen, deutet aber auch auf die innere Hierarchisierung einer großen Fraktion hin - und bedeutet für jene, die Interesse an den Professionalisierungsprozess zeigen, neue Ansätze für eine Analyse.

\subsection{MSZP - zusammengeschrumpfte Fraktion}

Infolge der Parlamentswahlen 2010 schrumpfte die Fraktion der Ungarischen Sozialistischen Partei verglichen mit den früheren Jahren auf ein Drittel zusammen. Am Ende der letzten Wahlperiode gehörten ihr 188 Abgeordnete an, die Zahl sank im neu gewählten Parlament auf 59. Verglichen mit den früheren Ergebnissen - besonders mit dem Spitzenwert von 209 Abgeordneten 1994 - stellt dies tatsächlich eine drastische Veränderung dar und kommt den 33 Mandaten des Jahres 1990 gefährlich nahe.

Die Änderung, die am meisten ins Auge sticht, ist die geringe Anzahl von neuen Abgeordneten. Nur sechs Parlamentarier gehören in diese Gruppe, gut zehn Prozent und damit circa ein Viertel jenes Wertes, der bei FIDESZ und KDNP zu finden ist ( 42 beziehungsweise 44 Prozent). Dies ist natürlich im Spiegel der Wahlergebnisse verständlich: Politiker, die wichtig für die Partei sind, beziehungsweise jene, die eine starke Hausmacht besitzen, kamen auf die aussichtsreichen Plätze der territorialen Listen und der Landesliste, während die neuen Gesichter von weniger vorteilhaften Positionen in die Wahlen gingen. Dies ist auch deshalb wichtig, weil die MSZP nur in zwei Einzelwahlkreisen ein Mandat erreichen konnte (beide in Budapest) und damit die Listenplätze besonders aufgewertete wurden. (Man darf aber nicht vergessen, dass die „ungebrauchten“ Stimmen auf der anderen Seite die Zahl der Listenmandate erhöhte.) So verständlich es ist, dass Spitzenpolitiker an ihren Mandaten hängen, so bedeutet dies aber zugleich das - auch in der Selektion sichtbar werdende - Ausbleiben der Erneuerung oder mindestens ihre Abschwächung im Parlament. (Für die letzte Wahlperiode konnte etwas Ähnliches im Falle von SZDSZ und MDF diagnostiziert werden.) Es geht hier natürlich um eine heikle politische Frage: Man müsste - auch bei der Selektion - sich so erneuern, dass weder das Profil noch die Gesichter der Partei verloren gehen. Man müsste sowohl die Kontinuität als auch die Veränderung im immer enger wer-

16 Den Großteil dieser Personen machen jene Minister und Staatssekretäre aus, die zugleich Abgeordnete sowie Mitglied der Fraktionsführung und/oder im Parlamentspräsidium sind. 
denden politischen Raum zur Geltung bringen. Es hat den Anschein, dass in solch einer Situation die Erneuerung des Profils und der Selektion unumgänglich ist. Für die hierbei zu Tage tretende Ambivalenz der Sozialistischen Partei ist kennzeichnend, dass die Niederlage Frauen mehr traf, da ihr Anteil von 13,3 Prozent in der vorangegangenen Wahlperiode auf 8,5 Prozent sank. Die in der Partei vorgeschriebene 20-Prozentquote wurde auch früher nie erfüllt. Nach den Wahlen des Jahres 2010 verblieben nur die mit den „alten“ Gesichtern gleichbedeutenden professionellen Politikerinnen in den Sitzreihen der MSZP.

Vergleicht man die MSZP-Fraktion mit jener des FIDESZ, so finden sich immer noch mehr Gemeinsamkeiten als zwischen den Sozialisten und den beiden neuen Parteien (LMP und Jobbik), wenn es um folgende Variablen geht: Frauenanteil, in Budapest geboren, Hochschulabschluss, Anteil der verschiedenen Diplome. Aber auch die Unterschiede sind gut sichtbar. Im Kreis der sozialistischen Abgeordneten ist der Anteil der Ökonomen doppelt so hoch wie bei FIDESZ, und in ihrem Kreis sind kaum Ärzte zu finden.

Eine weitere Konsequenz des Zusammenschrumpfens der MSZP-Fraktion ist der hohe Anteil von Abgeordneten, die Positionen in der Parteiführung bekleiden (46 Prozent). Zwischen 2006 und 2010 gehörten von 196 Abgeordneten 59, also 30 Prozent, in diese Gruppe. Unter den Parteichefs der Komitate sind nur zwei nicht im Parlament ${ }^{17}$, und zwar weil sie auf der fünften beziehungsweise siebten Stelle der Territoriallisten - also auf verlorenen Posten - standen. Fünfundzwanzig Abgeordnete waren als Ministerpräsident, Minister oder Staatssekretär Mitglieder der sozialistischen Gyurcsány- und Bajnai-Regierung (42 Prozent). Damit hatten in der Summe etwa 80 Prozent der Mitglieder der sozialistischen Parlamentsfraktion früher einen Regierungsposten oder eine Funktion in der Parteiführung inne. (Diese Zahl erinnert gespenstisch an die Zusammensetzung der letzten SZDSZ-Fraktion.) Abgeordnete, die bloß eine Vergangenheit als „einfaches“ Mitglied des Parlaments aufweisen, gibt es kaum.

Davon abgesehen zeigt die MSZP-Fraktion große Ähnlichkeiten zu früheren Wahlperioden. Zwar ist der Anteil der Frauen zurückgefallen, das Durchschnittsalter hat sich aber kaum verändert (von 50,7 auf 49,4 Jahre); wegen der geringen Erneuerungsrate ist die Kennziffer, wie oft die Mitglieder der Fraktion gewählt wurden, bedeutend gestiegen (von 2,5 auf 3,4) und der Anteil der in Budapest Geborenen hat sich etwas vergrößert (von 20,9 Prozent auf 23,7 Prozent). Aber weder bei dem Anteil der Hochschulabsolventen, noch dem Studienfach gibt es bedeutende Änderungen - lediglich der Anteil an Ärzten und jener mit anderen Berufen ging zurück. Zusammenfassend kann man feststellen, dass die MSZPFraktion im Großen und Ganzen aus den Vertretern der Parteielite besteht.

\subsection{Die Christdemokraten - spezifisches Profil}

Die Fraktion der KDNP befindet sich in einer eigenartigen Situation: Es passiert nicht zum ersten Mal, dass die Partei nicht als selbstständige Kraft bei den Wahlen antritt, danach aber doch eine eigene Fraktion bildet und sogar an der Regierung beteiligt wird. Diese Tatsache wird jenen Wissenschaftlern nicht wenig Kopfzerbrechen verursachen, die an Hand der traditionellen Kriterien der Koalitionstheorie dieses Bündnis erklären wollen. Ein Teil

17 Ungarn besteht aus 20 großen Verwaltungseinheiten: 19 Komitate und die Hauptstadt Budapest. 
dieser sonderbaren Situation ist auch, dass einige Abgeordnete der FIDESZ nahestehen, manchmal sogar eine doppelte Parteimitgliedschaft besitzen. ${ }^{18}$

Die KDNP-Fraktion war noch nie so groß wie jetzt (36 Personen), auch damals nicht, als die Christdemokraten als eigenständige Partei in die Wahlen gingen (1990 und 1994: 22 Abgeordnete, 2006: 26 Abgeordnete); die Wahlkoalition wirkt sich also für die Partei vorteilhaft aus. Der Anteil der neuen Abgeordneten ist höher als bei FIDESZ, was aber in diesem Fall zu keiner Verjüngung führte: Die Christdemokraten blieben wie früher die Fraktion mit dem höchsten Durchschnittsalter im Parlament. Nicht nur die Erneuerungsraten ähneln sich bei KDNP und FIDEZ, sondern auch die durchschnittliche Mandatsdauer ist gleich, nämlich 2,3 Wahlperioden (siehe Tabelle 5); dieser Wert wird nur von den Sozialisten überboten $(3,4)$. Dies deutet auf den doppelten Charakter der KDNP-Fraktion hin: Einerseits findet man in ihren Reihen lang gediente Abgeordnete, die früher auch Regierungsposten innehatten und der Partei ihr Antlitz geben. Die lange Mandatsdauer ist auch dadurch zu erklären, dass die Politiker der KDNP, die 1998 den Einzug ins Parlament verpassten, FIDESZ beitraten und so weiterhin Abgeordnete blieben.

Hinsichtlich der Schulabschlüsse ist die KDNP-Fraktion die höchstgebildete; ihr gehören auch Abgeordnete - entsprechend dem politischen Profil der Partei - an, die theologische Hochschulen absolviert haben. Auch der Anteil der Juristen ist in dieser Fraktion der höchste. Verglichen mit der KDNP-Fraktion des Jahres 2006 ist die Abnahme des Frauenanteils bemerkenswert (von 8,3 auf 5,6 Prozent) und der Anstieg der Mitglieder der Parteiführung (von 30,4 auf 38,9 Prozent).

Zusammenfassend ist festzustellen, dass die Fraktion der KDNP sich in vieler Hinsicht von den anderen Fraktionen unterscheidet, FIDESZ inbegriffen. Das spezifische Profil ergibt sich einerseits aus dem Elitencharakter, andererseits durch die Vertretung traditioneller Werte. Die Frage, in welcher Weise dieses eigenständige Fraktionsprofil als Parteiprofil erscheinen und in Anziehungskraft in der Wählerschaft transformiert werden kann, zeigt weit über diesen Beitrag hinaus.

\subsection{Jobbik - jünger, männlicher, weniger gebildet}

Die Bewegung Jobbik Magyarországért trat - nachdem sie erfolgreich die Hürde bei den EP-Wahlen im Jahre 2009 überwinden konnte ${ }^{19}$ - 2010 erstmals allein an. Die politische Formation kann auf eine etwa zehnjährige Vergangenheit zurückblicken, da sie als Jobboldali Ifjúsági Közösség (Jobbik / Rechte Jugendgemeinschaft) 1999 gegründet wurde. Im Jahre 2003 wurde aus der Jugendorganisation eine Partei. Bei den nächsten Parlamentswahlen bildete Jobbik mit MIÉP eine gemeinsame Liste, die 2,2 Prozent erhielt. Damit übersprang sie die Einprozentgrenze und hatte so Recht auf staatliche finanzielle Unterstützung; der Einzug ins Parlament schlug aber fehl. Die Kooperation zwischen den beiden Parteien wurde später beendet, und Jobbik setzte ihre politische Arbeit allein fort.

Die Partei erhielt 201047 Mandate und bildet so die drittstärkste Fraktion im Parlament. Als neue Partei ist auch der überwiegende Teil der Abgeordneten neu, aber auch in

18 Bei den Websites einiger KDNP-Abgeordneter findet man einen Link zu FIDESZ. Es ist möglich, dass es sich hier nur um eine organisatorische Frage handelt; zugleich hat dies aber Signalwirkung.

19 Jobbik erhielt drei der 22 ungarischen Mandate. 
dieser Fraktion finden sich drei Rückkehrer. Diese gehörten zwischen 1998 und 2002 - wenig verwunderlich - der MIÉP an. Damit ist die Frage aufgeworfen, wie eng eigentlich die Beziehung zu MIÉP ausgeprägt ist. Wie viele Jobbik-Parlamentarier begannen ihre politische Karriere in der Partei der „Ungarischen Wahrheit und des Lebens“? Eine Durchsicht der Lebensläufe zeigt, dass zwölf Personen (25 Prozent) ihre Mitgliedschaft in MIÉP erwähnen, eine Person war Mitglied der Jugendorganisation, zwei Personen vermerken ihre frühere Sympathie mit MIÉP. Mindestens ein Viertel der Jobbik-Abgeordneten war also früher in MIÉP tätig. Die Lebensläufe erwähnen aber auch eine Reihe anderer Organisationen, die vor allem der ungarischen rechtsextremen Subkultur zuzurechnen sind. Einige Abgeordnete schreiben auch über ihre Verbindung zur Ungarischen Garde.

Neben einer Vergangenheit in der MIÉP gibt es in der Jobbik-Fraktion aber auch Mitglieder, die früher in anderen im Parlament vertretenen Parteien aktiv waren. Hier werden MDF, KDNP, FKGP oder sogar FIDESZ genannt. ${ }^{20}$ Einige kommen aus Umweltorganisationen. Damit zeigt Jobbik ein ganz anderes Bild als die aus verschiedenen politischen Plattformen bestehenden, aber doch als Mainstream betrachteten FIDESZ und MSZP: Es geht hier um „neue Politiker“, die in verschiedenen Bewegungen und Zivilorganisationen ihren Platz suchen, während in den alten Parteien die formale Kommunal- und Parteipolitik den Hintergrund der Abgeordneten ausmachen.

Jobbik wurde - wie schon erwähnt - 2003 in eine Partei umgeformt. Damals schlossen sich acht der heutigen Fraktionsmitglieder der Partei an - unter ihnen der heutige Parteiführer Gábor Vona. Die nächste große Eintrittswelle bei den Mitgliedern der Fraktion fand 2007 und 2008 statt: Insgesamt 18 Personen vermerken dieses Jahr als Eintrittsdatum in ihrem Lebenslauf. Aber noch im Jahre 2009 traten einige der ein Jahr später ins Parlament Gewählten in die Partei ein. Das heißt, neben den „Alten“ des Jahres 2003 traten 2010 auch solche als Kandidaten bei Jobbik an, die erst seit ein bis zwei Jahren Parteimitglieder waren. Dies ist aber für eine junge Partei nicht verwunderlich, wenn bei Parlamentswahlen viele neue Kandidaten nötig sind und diese aus dem eigenen Kader nicht leicht aufzufüllen sind.

Ein Vergleich der Abgeordneten der Jobbik mit dem ganzen Parlament zeigt die folgenden Unterschiede, die das Profil der Fraktion bestimmen:

- das Durchschnittsalter ist niedriger (ganzes Parlament: 47 Jahre, Jobbik: 41 Jahre);

- der Frauenanteil ist geringer (9,4 zu 6,4 Prozent);

- der Anteil der in Budapest Geborenen ist um ein Drittel höher (27 zu 36 Prozent);

- der Anteil der Hochschulabsolventen ist geringer (87 zu 70 Prozent), in der Fraktion haben sieben Personen nur Mittelschulabschluss, vier weitere waren bei der Wahl ins Parlament Studenten;

- unter jenen mit Hochschulabschluss ist der Anteil der Juristen niedriger (24 zu 15 Prozent).

\subsection{LMP - ein neuer Anfang?}

Wenn eine Partei zum ersten Mal in die parlamentarische Arena tritt - und besonders wenn es sich um eine Parteineugründung handelt - kann man mit Recht die Frage aufwerfen, welche Wirkung die Gründung der Partei und ihr Organisationsmodus auf das Profil der 
Abgeordneten ausübt. In Transformationsländern wie Ungarn kommt ein weiterer Gesichtspunkt hinzu: Zeigt die Fraktion vielleicht Ähnlichkeiten mit den damals neuen Parteien bei den Wahlen 1990?21 Lehet Más a Politika wurde 2003 gegründet, aber erst bei den EP-Wahlen 2009 einer breiteren Öffentlichkeit bekannt.

2010 gelangten ihre Abgeordneten - wie schon erwähnt - nur über Listenplätze ins Parlament; den meisten fehlte eine lokale Verankerung - man könnte auch sagen ihre lokale Bekanntheit. ${ }^{22}$ Ein Mitglied der Fraktion war zwischen 1990 und 1994 für die MDF im Parlament.

Die mangelnde lokale Verankerung bedeutet aber nicht das Fehlen einer früheren politischen Tätigkeit: Im Hintergrund der Erfahrungen der Abgeordneten finden sich Umwelt-, Menschenrechts- und Jugendorganisationen, sogar internationale Organisationen. Die schnelle und neue Techniken (Internet) in Anspruch nehmende Organisation der Partei spiegelt sich im hohen Anteil der in Budapest Geborenen wider. Zwei Drittel der Mitglieder der Fraktion gehören in diese Gruppe. Damit übersteigt sie bei weitem die im Allgemeinen als Budapester Partei betrachtete SZDSZ (in der letzten Wahlperiode betrug deren Anteil der in Budapest Geborenen 40 Prozent). Zweifellos ist LMP zurzeit die jüngste Partei im Parlament, ist aber älter als FIDESZ im Jahre 1990, deren Abgeordnete damals sämtlich unter 35 waren. Ein weit wichtigerer Unterschied zu FIDESZ ist, dass der Frauenanteil in der Fraktion (wegen der angewandten Methode der Kandidatenaufstellung) 31 Prozent ausmacht. LMP ist seit 1990 die erste Partei der ungarischen Demokratie, die die inhaltliche Chancengleichheit der Geschlechter zueigen machte und diese auch zur Geltung brachte. Das Profil ist eindeutig: Städtisch-hauptstädtische, junge Intellektuelle machen die Basis der Partei aus. Dass bloß 81 Prozent eine Hochschulbildung aufweisen (und somit formal nur noch von Jobbik unterboten), ist irreführend. Zwei Personen haben zwar ihr Studium abgeschlossen, aber kein Diplom, unter ihnen der landesbekannte Dichter Endre Kukorelly. Die Mitglieder der Fraktion besitzen eine ausgesprochen hohe Bildung, mitunter auch mit internationalen Erfahrungen. Dennoch ist fraglich, inwieweit diese kleine Fraktion mit 16 Mitgliedern den parlamentarischen Angelegenheiten ihren eigenen Stempel aufdrücken kann.

\section{Weitere Aspekte}

\subsection{Frauen und Männer}

Ein äußerst sensibles Gebiet und wichtigster Beweis für die Ungleichheit zwischen Frauen und Männern sind politische Positionen, im gegebenen Fall die unterschiedliche Stabilität der parlamentarischen Mandate zwischen den beiden Geschlechtern. Ohne Wiederwahl kann der Beruf des Politikers nicht ausgeübt werden. Wenn die Wiederwahl ausbleibt, ist dies ein Fiasko des Politikers oder der Politikerin, und wenn in diesem Zusammenhang ein signifikanter Geschlechterunterschied diagnostiziert wird, kann von einem geschlechtsspe-

21 Hier denken wir an FIDESZ, MDF und SZDSZ und nicht an die so genannten historischen Parteien, die schon vor 1947 existierten oder an die Nachfolgepartei MSZP.

22 Die lokale Verankerung erscheint gerade bei dem Abgeordneten, einem Bürgermeister, der seitdem die Fraktion schon verlassen hat. 


\begin{tabular}{|c|c|c|c|c|c|}
\hline & & $\begin{array}{c}\text { Neue } \\
\text { Abgeordnete }\end{array}$ & $\begin{array}{c}\text { Wiedergewählte } \\
\text { Abgeordnete }\end{array}$ & Rückkehrer & Insgesamt \\
\hline \multirow{2}{*}{ Männer } & Anzahl & 154 & 179 & 18 & 351 \\
\hline & $\%$ & 43,9 & 51,0 & 5,1 & 100 \\
\hline \multirow{2}{*}{ Frauen } & Anzahl & 21 & 14 & 0 & 35 \\
\hline & $\%$ & 60,0 & 40,0 & 0 & 100 \\
\hline \multirow[t]{2}{*}{ Insgesamt } & Anzahl & 175 & 193 & 18 & 386 \\
\hline & $\%$ & 45,3 & 50,0 & 4,7 & 100 \\
\hline
\end{tabular}

zifischen Erfolg oder Fiasko gesprochen werden. Schon frühere Studien haben auf diese Problematik hingewiesen und festgestellt, dass Frauen eher aus dem Parlament herausfallen und ihre Mandatszeit insgesamt kürzer ist. ${ }^{23}$ Dieses Phänomen wurde auch durch die Wahlen 2010 in Ungarn eindeutig bestärkt.

Aus Tabelle 7 geht hervor, dass ein signifikanter Unterschied zwischen Frauen und Männern bei der Stabilität des Mandats besteht. In der 2010 beginnenden Wahlperiode sind etwa 60 Prozent der Frauen zum ersten Mal im Parlament, während dieser Wert bei den Männern nur 44 Prozent ausmacht. Dies steht natürlich auch in Zusammenhang mit den Veränderungen der Parteienlandschaft: Die Verliererpartei MSZP bewies erneut, dass sie als linke Partei die Chancengleichheit für Frauen gering schätzt, denn unter den Rahmenbedingungen der immer geringer werdenden Wahlchancen selektierte sie die Frauen aus; die neue LMP hingegen brachte durch ihre Frauenquote mehr Kandidatinnen ins Parlament.

Im Zusammenhang mit der geschlechterspezifischen Zusammensetzung des Parlaments fällt auch auf, dass alle Rückkehrer Männer sind. Teilweise erklärt sich dies daraus, dass die meisten Rückkehrer in den Reihen von FIDESZ und Jobbik zu finden sind. Daneben müssen aber auch die zur Rückkehr nötigen Ressourcen und, wie es den Anschein hat, deren gender-spezifischer Inhalt in Rechnung gestellt werden. Aus der schon oben zitierten Studie von Gyömöre ist bekannt, dass neben der Gesamtleistung der Partei die günstige Position bei der Kandidatenaufstellung sowie die im Karriereweg sich widerspiegelnden Ressourcen für die Rückkehr eine wichtige Rolle spielen. ${ }^{24}$ Hinzu kommt die Annahme, dass sich Frauen vielleicht früher aus der Politik zurückziehen, weniger zur Pflege des Karriereweges entschließen und sich weniger einer erneuten Probe aussetzten wollen.

Aus Tabelle 8 sind in Hinsicht der politischen Rollen - und Möglichkeiten - die Unterschiede zwischen Frauen und Männer ersichtlich. Seit 1990 ist es ohne Beispiel, dass sich keine Frau unter den Ausschussvorsitzenden findet. In den ersten drei Wahlperioden war je eine Frau in einer solchen Position, in der vierten und fünften haben jeweils vier Frauen einem Ausschuss vorgesessen. ${ }^{25}$ Diese Situation wird auch nicht durch die weniger politi-

23 Siehe Gabriella Ilonszki, Konszolidáció vagy bezárkózás? A 2006-ban megválasztott parlamenti képviselők jellemzői, in: Gergely Karácsony (Hrsg.), Parlamenti választás, Budapest 2006, S. 229 - 252; Gabriella Ilonszki / Várnagy Réka, a.a.O. (Fn. 7).

24 Vgl. Máté Gyömöre, a.a.O. (Fn. 14), S. 122 f.

25 Vgl. Ida B. Kelemen, Cherchez la femme!, in: Gabriella Ilonszki (Hrsg.), a.a.O. (Fn. 5), S. 67 94. 


\begin{tabular}{|l|r|r|r|r|c|}
\hline Tabelle 8: Politische Positionen der weiblichen und männlichen Abgeordneten im Parlament \\
\hline & \multicolumn{2}{|c|}{$\begin{array}{c}\text { Frauen } \\
(\mathrm{N}=35)\end{array}$} & \multicolumn{2}{c|}{$\begin{array}{c}\text { Männer } \\
\text { (N=351) }\end{array}$} & $\begin{array}{c}\text { Statistischer } \\
\text { Zusammenhang } \\
\text { (Phi) }\end{array}$ \\
\cline { 2 - 6 } & $\mathrm{N}$ & $\%$ & $\mathrm{~N}$ & $\%$ & .006 \\
\hline Ausschussvorsitz & - & - & 19 & 5,4 & .006 \\
Unterausschussvorsitz & 10 & 28,6 & 34 & 9,7 & nicht signifikant \\
Parlamentsfunktion & 1 & 2,9 & 15 & 4,3 & nicht signifikant \\
Führende Position in der Partei & 14 & 40,0 & 121 & 34,5 & .019 \\
Position in der Kommunalpolitik zur & 13 & 37,1 & 203 & 57,8 & .062 \\
Zeit der Wahl & 3 & 8,6 & 75 & 21,4 & \\
Bürgermeister zur Zeit der Wahl & 3 & \\
\hline \multicolumn{7}{|l|}{ Quelle: Ungarische Abgeordneten-Datenbank. }
\end{tabular}

sche Ressourcen bereithaltenden führenden Positionen in Unterausschüssen kompensiert. Lediglich bei den höheren Ämter in der Partei weisen die weiblichen Abgeordneten einen höheren Anteil als ihre männlichen Kollegen auf, aber auch hier ist das Maß nicht signifikant (vgl. Tabelle 8). Hier bestätigt sich wieder, dass in der Karriere von Politikerinnen die Partei eine wichtigere Rolle spielt - anders formuliert: Die anderen Ressourcen sind weniger vorhanden, und Frauen haben nur dann eine Chance, in der durch die Parteien gesteuerten Selektion in eine günstige Position zu gelangen, wenn sie in der Partei vorwärts gekommen sind. Die Parteien sind die wichtigsten Instanzen für die Selektion. Dies gilt insbesondere für das Erlangen eines Parlamentsmandates, wie dies in der internationalen ${ }^{26}$ und auch der ungarischen Literatur festgestellt wurde. ${ }^{27}$

Die Schranken anderer Ressourcen zeigen sich auch im niedrigeren Anteil bei den kommunalpolitische Posten oder bei der Funktion als Bürgermeister, obwohl - wie gezeigt gerade diese Positionen eine der Quellen für die Wahl und besonders die Wiederwahl bedeuten.

\subsection{Langdienende Abgeordnete}

Die Attribute der lang dienenden Abgeordneten beschäftigten mit Recht sowohl Wissenschaftler als auch die Wähler, weil sie nicht zuletzt Auskunft darüber geben, was die Basis der Machterhaltung ausmacht.

Durch die Wahlen im Jahre 2010 kam es zu einem Austausch von etwa der Hälfte der Mitglieder des Parlaments (45 Prozent neue Abgeordnete und fünf Prozent Rückkehrer). Unter den „Altgedienten“ befinden sich nur noch neun Personen, die bereits 1990 ins Parlament gewählt wurden, und zwar neun Männer. Den letzten großen Schub für das Verschwinden der Abgeordneten der ersten Stunde besorgte die letzte Wahl: Erstens sind MDF und SZDSZ nicht mehr im Parlament vertreten; in der vergangenen Wahlperiode

26 Vgl. Miki Caul Kittilson, Women's Representation in Parliament. The Role of Political Parties, in: Party Politics, 5. Jg. (1999), H. 1, S. 79 - 98.

27 Vgl. Gabriella Ilonszki, The Impact of Early Party Consolidation on Female Representation and the Mixed Electoral System in: Manon Tremblay (Hrsg.), Women and Legislative Representation. Electoral Systems, Political Parties, and Sex Quotas, New York 2008, S. 205 - 218. 
fanden sich unter ihnen noch elf Personen, die seit 1990 im Parlament saßen. Zweitens ist die Fraktion der MSZP stark geschrumpft; vor 2010 gab es hier noch 13 Abgeordnete, die schon im ersten Parlament dabei waren. Aber auch aus der ersten FIDESZ-Fraktion fehlen viele Namen. Einige wechselten die Partei, andere schieden endgültig aus der Politik aus, andere sind zwar immer noch politisch aktiv, aber im Europäischen Parlament tätig. Von den ursprünglichen 22 sind nur noch fünf Mitglieder des Parlaments (unter ihnen der amtierende Ministerpräsident Viktor Orbán und der Parlamentspräsident László Kövér). ${ }^{28}$

Auch die Zahl derer, die zum fünften Mal ins Parlament gewählt wurden ist gering: Es handelt sich um 24 Abgeordnete, das heißt circa sechs Prozent des Parlaments. Hier ist das Verhältnis zwischen den Parteien etwas anders: 15 gehören zu den Sozialisten (als späten Ausdruck des großen Sieges von 1994), und nur sieben sitzen in der FIDESZ-Fraktion, zwei weitere bei den Christdemokraten. Der große FIDESZ-Sieg von 1998 schlägt sich in der nächsten Gruppe nieder, die zum vierten Mal ins Parlament einziehen konnten: In der FIDESZ-Fraktion gehören 47 Personen zu ihr.

Im sechsten ungarischen Parlament beträgt die durchschnittliche Wahlzahl 2,3, also mehr als drei Viertel (76 Prozent) der Abgeordneten erhielten ihr Mandat 2010 beziehungsweise bei den zwei vorhergehenden Parlamentswahlen.

\subsection{Ungarische Abgeordnete im Vergleich}

Um die vorgestellten Ergebnisse besser einordnen zu können, ist ein Blick auf Daten von Ländern in der Umgebung Ungarns nützlich. Besonders Polen und Tschechien bieten sich an, weil sie in der Entwicklung des Parteiensystems ähnliche Tendenzen bei der Bipolarität und der effektiven Parteienzahl aufweisen. In Ungarn kippte zwar das bipolare parteipolitische Feld im Jahre 2010; dennoch bleibt der Überblick im Zeitvergleich relevant.

Die ungarischen Abgeordneten weisen weit häufiger Hochschulabschlüsse auf als die Parlamentarier in Polen und Tschechien, und dies gilt auch noch nach dem Rückgang der Hochschulabsolventen 2010. Interessant ist auch das Stagnieren des Frauenanteils in Ungarn, während in Polen und Tschechien die Dynamik - unabhängig davon, ob es hinunter oder hinauf geht - mit den Wahlergebnissen und/oder dem Wahlsystem zusammenhängt.

Sehr augenfällig ist der unterschiedliche Trend bei den wiedergewählten Abgeordneten in Ungarn: Das „Einfrieren“ der politischen Elite war bis 2010 hier eindeutig. In Tschechien kann man Stagnation konstatieren; der Anteil der Wiedergewählten sank erst bei den Wahlen 2010. Der polnische Fall gestaltete sich, wenn auch mit geringerer Intensität, parallel zum ungarischen, aber nach den Wahlen 2005 und 2007 ging der Anteil der Abgeordneten mit längerer Mandatsdauer weiter zurück und stabilisierte sich unter 50 Prozent. Trotz der bedeutenden Umstrukturierung des Parteisystems in Ungarn im Jahre 2010 sind hier immer noch am meisten „Altgediente“ anzutreffen (vgl. Tabelle 9).

28 In der Fraktion der MSZP finden sich nur zwei Abgeordnete, die schon dem ersten demokratisch gewählten Parlament angehörten. 


\begin{tabular}{|l|l|c|c|c|c|}
\hline \multicolumn{3}{|c|}{ Tabelle 9: Eigenschaften der Parlamentsabgeordneten in Polen, Tschechien und Ungarn } \\
\hline \multirow{4}{*}{ Land } & Wahlperiode & $\begin{array}{c}\text { Anteil } \\
\text { der Frauen (\%) }\end{array}$ & $\begin{array}{c}\text { Durchschnitts- } \\
\text { alter }\end{array}$ & $\begin{array}{c}\text { Hochschul- } \\
\text { abschluss (\%) }\end{array}$ & $\begin{array}{c}\text { Anteil der } \\
\text { Wiedergewählten } \\
(\%)\end{array}$ \\
\hline \multirow{4}{*}{ Polen } & $2001-2005$ & 20,2 & 48,2 & 80,7 & 45,4 \\
& $2005-2007$ & 20,2 & 49,2 & 76,7 & 58,3 \\
& $2007-$ & 20,4 & 47,8 & 92,8 & 65,7 \\
\hline \multirow{4}{*}{ Tschechien } & $1998-2002$ & 15,0 & 45,0 & 74,5 & 57,0 \\
& $2002-2006$ & 17,0 & 46,9 & 81,0 & 57,0 \\
& $2006-2010$ & 15,0 & 49,0 & 78,5 & 55,5 \\
& $2010-$ & 22,0 & 47,2 & 82,0 & 43,0 \\
\hline \multirow{5}{*}{ Ungarn } & $1998-2002$ & 7,8 & 46,3 & 92,9 & 50,9 \\
& $2002-2006$ & 9,7 & 46,7 & 93,9 & 66,0 \\
& $2006-2010$ & 10,6 & 49,6 & 91,9 & 71,4 \\
& $2010-$ & 9,1 & 47,3 & 87,0 & 54,4 \\
\hline
\end{tabular}

\section{Abschottung der parlamentarischen Elite?}

Es scheint, als ob sich mit den Wahlen 2010 ein großer Wandel im Parlament vollzieht, der die Prozesse der letzten 20 Jahre abschließt. Diese Annahme trifft zu, wenn die Veränderungen im Parteiensystem, die Umstrukturierung des Regierungssystems und durchaus auch konkrete Politikfelder untersucht werden. Das Profil der Abgeordneten deutet jedoch auf die fortgesetzte Zentralisierung, Homogenisierung und Ämterhäufung sowie Positionsverflechtungen hin. Neben einer gewissen personellen Erneuerung lebt das Phänomen der Konzentrierung und des „Einfrierens der Elite“ weiter. Trotz der Veränderungen der Parteien sind wir Augenzeugen einer Abschottung der parlamentarischen Elite. Die Zahl der Kandidaten nahm bei den Wahlen 2010 weiter ab: Von 1.623 im Jahr 1990 sank sie auf 814 (1994: 1.874; 1998: 1.609; 2002: 1.204; 2006: 995). Dennoch kann strikt genommen erst einmal nur eine Abschottung hinsichtlich der formalen soziostrukturellen beziehungsweise soziodemographischen Daten behauptet werden.

Das allgemeine Antlitz des Parlaments wird verständlicherweise von FIDESZ bestimmt. Dadurch verstärken sich schon früher existierende Selektionsgesichtspunkte: Der Anteil an Abgeordneten mit einem kommunalpolitischen Hintergrund wuchs weiter, genauso wie die Mandatshäufung. ${ }^{29}$ Der hohe Anteil von Mitgliedern der Parteiführung ist typisch für jede

29 Auf diesen Aspekt kann hier nicht weiter eingegangen werden. Unter Mandatshäufung ist zu verstehen, dass zum Beispiel der Parlamentsabgeordnete Mitglied einer kommunalen Selbstverwaltung in einem Budapester Bezirk und zugleich in der hauptstädtischen Selbstverwaltung tätig ist - ähnliches gilt natürlich auch für andere Städte und die Komitatsebene. 
Fraktion und mit Ausnahme der beiden neuen auch die Ämterhäufung. Die Deselektion der Frauen geht weiter. Das extreme Beispiel der Abschottung ist die MSZP-Fraktion.

Diese Eigenschaften der Abgeordneten und Fraktionen wirken sich auf die Prozesse innerhalb des Parlaments und auf das Verhalten der Abgeordnete aus. Wie dies geschieht, ob und wie sich etwa die Abschottung, die aus den soziostrukturellen Daten spricht, in die konkrete Ausübung des Mandats fortsetzt, bedarf weiterer Untersuchungen. Darin wäre zum Beispiel zu fragen, mit welcher Kraft sich Abgeordnete, die Positionen häufen, überhaupt der Parlamentsarbeit widmen können oder wollen. Wie schon früher an anderer Stelle formuliert: Unter den Abgeordneten sind die professionellen Politiker nicht unbedingt professionelle Abgeordnete. ${ }^{30}$ Sie müssen sich anderswo behaupten, und es ist nicht klar, ob die Erfüllung ihrer Pflichten, die aus ihrem Mandat erwachsen, ihre erste Präferenz ist. Wenn aber die wichtigsten Akteure der Politik nicht viel vom Parlament verlangen und damit zufrieden sind, dass das Parlament eines unter vielen Gebäuden der Demokratie darstellt (und nicht ihr Kernstück), wird diese Situation zu keinem Rollenkonflikt führen. Sie kann aber gravierende Konsequenzen für die Politische Kultur und die Akzeptanz der parlamentarischen Demokratie und damit für das gesamte politische System nach sich ziehen.

30 Vgl. Sándor Kurtán / Gabriella Ilonszki, Munka, foglalkozás, hivatás - a képviselői professzionalizáció többszempontú elemzése, in: Gabriella Ilonszki (Hrsg.), a.a.O. (Fn. 5), S. 17 - 42, S. 41. 